\title{
Insight into the Narrow Structure in $\eta$ Photoproduction on the Neutron from Helicity-Dependent Cross Sections
}

L. Witthauer, ${ }^{1}$ M. Dieterle, ${ }^{1}$ S. Abt, ${ }^{1}$ P. Achenbach, ${ }^{2}$ F. Afzal,${ }^{3}$ Z. Ahmed, ${ }^{4}$ J. R. M. Annand,${ }^{5}$ H. J. Arends,${ }^{2}$ M. Bashkanov, ${ }^{6}$ R. Beck, ${ }^{3}$ M. Biroth, ${ }^{2}$ N. S. Borisov, ${ }^{7}$ A. Braghieri, ${ }^{8}$ W. J. Briscoe, ${ }^{9}$ F. Cividini, ${ }^{2}$ S. Costanza, ${ }^{8,}{ }^{,}$C. Collicott, ${ }^{10}$ A. Denig, ${ }^{2}$ E. J. Downie, ${ }^{2,9}$ P. Drexler, ${ }^{2}$ M. I. Ferretti-Bondy, ${ }^{2}$ S. Gardner, ${ }^{5}$ S. Garni, ${ }^{1}$ D. I. Glazier, ${ }^{5,6}$ D. Glowa, ${ }^{6}$ W. Gradl, ${ }^{2}$ M. Günther, ${ }^{1}$ G. M. Gurevich, ${ }^{11}$ D. Hamilton, ${ }^{5}$ D. Hornidge, ${ }^{12}$ G. M. Huber, ${ }^{4}$ A. Käser, ${ }^{1}$ V. L. Kashevarov, ${ }^{2}$ S. Kay, ${ }^{6}$ I. Keshelashvili, ${ }^{1, \dagger}$ R. Kondratiev, ${ }^{11}$ M. Korolija, ${ }^{13}$ B. Krusche, ${ }^{1,}$ A. B. Lazarev, ${ }^{7}$ J. M. Linturi, ${ }^{2}$ V. Lisin, ${ }^{11}$ K. Livingston, ${ }^{5}$ S. Lutterer, ${ }^{1}$ I. J. D. MacGregor, ${ }^{5}$ J. Mancell, ${ }^{5}$ D. M. Manley, ${ }^{14}$ P. P. Martel,,${ }^{2}$ V. Metag, ${ }^{15}$ W. Meyer, ${ }^{16}$ R. Miskimen, ${ }^{17}$ E. Mornacchi, ${ }^{2}$ A. Mushkarenkov, ${ }^{11,17}$ A. B. Neganov, ${ }^{7}$ A. Neiser, ${ }^{2}$ M. Oberle, ${ }^{1}$ M. Ostrick, ${ }^{2}$ P. B. Otte, ${ }^{2}$ D. Paudyal, ${ }^{4}$ P. Pedroni, ${ }^{8}$ A. Polonski, ${ }^{11}$ S. N. Prakhov, ${ }^{2,18}$ A. Rajabi, ${ }^{17}$ G. Reicherz, ${ }^{16}$ G. Ron,,${ }^{19}$ T. Rostomyan, ${ }^{1,}$ A. Sarty, ${ }^{10}$ C. Sfienti, ${ }^{2}$ M. H. Sikora, ${ }^{6}$ V. Sokhoyan, ${ }^{2,9}$ K. Spieker, ${ }^{3}$ O. Steffen, ${ }^{2}$ I. I. Strakovski, ${ }^{9}$ Th. Strub, ${ }^{1}$ I. Supek, ${ }^{13}$ A. Thiel, ${ }^{3}$ M. Thiel, ${ }^{2}$ A. Thomas, ${ }^{2}$ M. Unverzagt, ${ }^{2}$ Yu. A. Usov, ${ }^{7}$ S. Wagner, ${ }^{2}$ N. K. Walford, ${ }^{1}$ D. P. Watts, ${ }^{6}$ D. Werthmüller, ${ }^{1,5}$ J. Wettig, ${ }^{2}$ M. Wolfes, ${ }^{2}$ and L. Zana ${ }^{6}$

(A2 Collaboration at MAMI)

\author{
${ }^{1}$ Department of Physics, University of Basel, CH-4056 Basel, Switzerland \\ ${ }^{2}$ Institut für Kernphysik, University of Mainz, D-55099 Mainz, Germany \\ ${ }^{3}$ Helmholtz-Institut für Strahlen- und Kernphysik, University of Bonn, D-53115 Bonn, Germany \\ ${ }^{4}$ University of Regina, Regina, SK S4S OA2 Canada \\ ${ }^{5}$ SUPA School of Physics and Astronomy, University of Glasgow, Glasgow G12 8QQ, United Kingdom \\ ${ }^{6}$ SUPA School of Physics, University of Edinburgh, Edinburgh EEH9 3JZ, United Kingdom \\ ${ }^{7}$ Joint Institute for Nuclear Research, 141980 Dubna, Russia \\ ${ }^{8}$ INFN Sezione di Pavia, I-27100 Pavia, Italy \\ ${ }^{9}$ Center for Nuclear Studies, The George Washington University, Washington, DC 20052-0001, USA \\ ${ }^{10}$ Department of Astronomy and Physics, Saint Marys University, Halifax, Nova Scotia B3H 3C3, Canada \\ ${ }^{11}$ Institute for Nuclear Research, 125047 Moscow, Russia \\ ${ }^{12}$ Mount Allison University, Sackville, New Brunswick E4L 1E6, Canada \\ ${ }^{13}$ Rudjer Boskovic Institute, HR 10000 Zagreb, Croatia \\ ${ }^{14}$ Kent State University, Kent, Ohio 44242-0001, USA \\ ${ }^{15}$ II. Physikalisches Institut, University of Giessen, D-35392 Giessen, Germany \\ ${ }^{16}$ Institut für Experimentalphysik, Ruhr Universität, 44780 Bochum, Germany \\ ${ }^{17}$ University of Massachusetts Amherst, Amherst, Massachusetts 01003, USA \\ ${ }^{18}$ University of California at Los Angeles, Los Angeles, California 90095-1547, USA \\ ${ }^{19}$ Racah Institute of Physics, Hebrew University of Jerusalem, Jerusalem 91904, Israel
}

(Received 13 April 2016; published 22 September 2016)

\begin{abstract}
The double polarization observable $E$ and the helicity dependent cross sections $\sigma_{1 / 2}$ and $\sigma_{3 / 2}$ were measured for $\eta$ photoproduction from quasifree protons and neutrons. The circularly polarized tagged photon beam of the A2 experiment at the Mainz MAMI accelerator was used in combination with a longitudinally polarized deuterated butanol target. The almost $4 \pi$ detector setup of the Crystal Ball and TAPS is ideally suited to detect the recoil nucleons and the decay photons from $\eta \rightarrow 2 \gamma$ and $\eta \rightarrow 3 \pi^{0}$. The results show that the narrow structure previously observed in $\eta$ photoproduction from the neutron is only apparent in $\sigma_{1 / 2}$ and hence, most likely related to a spin-1/2 amplitude. Nucleon resonances that contribute to this partial wave in $\eta$ production are only $N 1 / 2^{-}\left(S_{11}\right)$ and $N 1 / 2^{+}\left(P_{11}\right)$. Furthermore, the extracted Legendre coefficients of the angular distributions for $\sigma_{1 / 2}$ are in good agreement with recent reaction model predictions assuming a narrow resonance in the $P_{11}$ wave as the origin of this structure.
\end{abstract}

DOI: 10.1103/PhysRevLett.117.132502

Photoproduction of $\eta$ mesons is important for the investigation of the nucleon excitation spectrum. Because of its isoscalar nature, the $\eta$ only couples to isospin $I=1 / 2 N^{\star}$ resonances. In the threshold region, this reaction is completely dominated by the excitation of the $N(1535) 1 / 2^{-}$resonance [1] and at higher incident photon energies, contributions from several other excited nucleon states have been identified [2]. Currently, a large 
effort is underway at modern photon-beam facilities (see Ref. [2] for a recent summary) to study the $\gamma p \rightarrow p \eta$ reaction using both single and double polarization observables. However, during the last few years, photoproduction of $\eta$ mesons off the neutron has attracted additional interest. The reason is the discovery of an unusually narrow structure in the excitation function at incident photon energies of $1 \mathrm{GeV}$ (corresponding to an $\eta$-neutron invariant mass of $W \approx 1.67 \mathrm{GeV}$ ). This structure was first observed by the GRAAL Collaboration [3] and confirmed by the CBELSA/TAPS Collaboration [4,5] in Bonn, and at LNS in Sendai [6]. Recent high-statistics measurements at the MAMI facility in Mainz with deuterium and ${ }^{3} \mathrm{He}$ targets [7-9] have extracted a position of the narrow structure of $W=(1670 \pm 5) \mathrm{MeV}$ with a width of only $\Gamma=(30 \pm 15) \mathrm{MeV}$. This structure is not observed in $\eta$ photoproduction off the proton [10]. The cross section of $\gamma p \rightarrow p \eta$ shows only a small dip at this energy $[2,10]$. However, recently, two narrow structures were observed in the beam asymmetry $\Sigma$ of Compton scattering of the proton [11]. One of these structures appears close to the above discussed peak in $\eta$ production off neutrons and the other at $W \approx 1.726 \mathrm{GeV}$. Meanwhile, a counterpart of the latter peak was also unambiguously identified in the cross section of the $\gamma n \rightarrow n \eta$ reaction [12].

The nature of these structures has not yet been established. The prominent peak observed in $\eta$ production off the neutron at $W \approx 1.67 \mathrm{GeV}$ has been discussed as a new narrow resonance (with exotic properties) [13-17]. It is currently listed in the Review of Particle Physics (RPP) [18] as a tentative $N(1685)$ state with unknown spin and parity. However, other works suggest coupled-channel effects of known nucleon resonances [19,20], or contributions from intermediate strangeness states [21] as the underlying cause. A fit [22] from the Bonn-Gatchina $(\mathrm{BnGa})$ group to the high statistics MAMI deuteron data [7,9] suggests an interference in the $J^{P}=1 / 2^{-}$partial wave between contributions from the well-known $N(1535)$ and $N(1650)$ resonances. Fits of these unpolarized data with the $\mathrm{BnGa}$ model including a narrow $P_{11}$-like $N(1685)$ resonance were seen as inferior [22].

The aim of the present work is to determine the relevant partial wave directly from experimental data. For this purpose, the double polarization observable $E$ was measured with a longitudinally polarized target and a circularly polarized photon beam. It is defined as [23]

$$
E=\frac{\sigma_{1 / 2}-\sigma_{3 / 2}}{\sigma_{1 / 2}+\sigma_{3 / 2}},
$$

where $\sigma_{1 / 2}$ and $\sigma_{3 / 2}$ are the helicity dependent cross sections with antiparallel or parallel photon and nucleon spin, respectively. Nucleon resonances with spin $J=1 / 2$ contribute only to $\sigma_{1 / 2}$, while states with spin $J \geq 3 / 2$ can also couple to $\sigma_{3 / 2}$. Hence, structures in the $S_{11}$ or $P_{11}$ partial waves appear only in $\sigma_{1 / 2}$, but not in $\sigma_{3 / 2}$. So far, in $\eta$ production, this observable has only been explored for the reaction with free protons [24], for which it turned out to be very powerful in restricting parameters of reaction model analyses.

The experiments were performed at the Mainz MAMI accelerator [25]. Circularly polarized tagged photons [26] were created via the bremsstrahlung process with longitudinally polarized $\left(P_{e} \sim 80 \%\right)$ electrons. The beam helicity was flipped once per second. The polarization of the electron beam was measured daily with Mott scattering (after the linac stage of the accelerator at electron energies of $3.65 \mathrm{MeV}$ ) and constantly monitored with Møller scattering of the high energy electrons from the bremsstrahlung radiator. The polarization of the photon beam was deduced from the energy-dependent polarization transfer factors given by Olsen and Maximon [27]. The deuterated butanol $\left(\mathrm{C}_{4} \mathrm{D}_{9} \mathrm{OD}\right)$ target was polarized in the longitudinal direction using dynamic nuclear polarization [28]. The target polarization was measured before and after data taking using an NMR measurement technique and was interpolated by an exponential function. Because of small inhomogeneities of the polarizing magnetic field, the target was not homogeneously polarized across its diameter for the initial beam times (so that the NMR measurements did not correctly reflect the polarization degree in the target area interacting with the beam). Therefore, results were renormalized to the final data taking period for which this problem was resolved.

The experimental setup combined the Crystal Ball (CB) [29] and TAPS [30] calorimeters with additional detectors for charged particle identification [31] and covered $98 \%$ of $4 \pi$. The photons from the $\eta$ decays (results from $\eta \rightarrow \gamma \gamma$ and $\eta \rightarrow 3 \pi^{0} \rightarrow 6 \gamma$ were consistent and have been averaged) and the recoil nucleons were detected and analyzed. The detector was identical to the setup used for the measurements with unpolarized targets which is discussed in detail in Refs. [8,9]. Also, all analysis procedures were identical to those described in these references. This includes the clean identification of $\eta$ production off quasifree nucleons, the Monte Carlo simulations of the detector response, and the reconstruction of final-state kinematics used to remove the effects from nuclear Fermi motion. The latter is essential for the investigation of narrow structures.

The only complication resulted from the contribution from nucleons bound in the unpolarized carbon (and oxygen) nuclei in the butanol target. This background contributes only in the denominator of Eq. (1). It was determined from a measurement with a carbon foam target (which had identical geometry and density to the butanol target) and subtracted. Both measurements (butanol and carbon target) were normalized absolutely to photon fluxes, target surface densities, and detection efficiencies.

The double polarization observable $E$ for $\eta$ mesons in coincidence with recoil protons and neutrons is shown in Fig. 1. The systematic uncertainty was estimated from the uncertainty of the target $( \pm 10 \%)$ and photon beam 


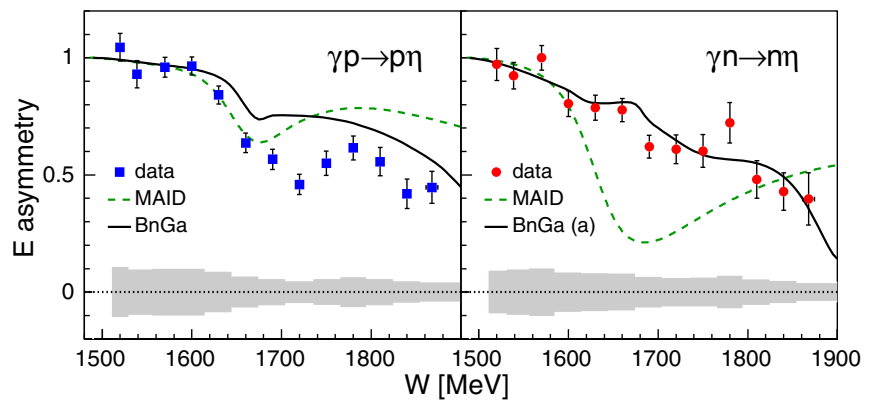

FIG. 1. Double polarization observable $E$ for $\gamma p \rightarrow p \eta$ (lefthand side) and $\gamma n \rightarrow n \eta$ (right-hand side). Gray shaded areas: systematic uncertainties. Curves: predictions from MAID (green, dashed) [32] and BnGa (model based on $S_{11}$ interference) [22] (black, solid line).

polarization $( \pm 2.7 \%)$. In addition, there is a small uncertainty related to the subtraction of the carbon background (all other uncertainties, e.g., from detection efficiencies, cancel to a large extent in the ratio of Eq. (1). This uncertainty was estimated from the precision of the photon flux measurements and the determination of the target surface densities. It is on the order of $2.5 \%$ and was added quadratically to the polarization degree uncertainties. As a cross check for the correct subtraction of the carbon background an analysis was done for which the denominator of the ratio in Eq. (1) was replaced by $2 \sigma_{0}$, where $\sigma_{0}$ is the unpolarized total cross section measured with a liquid deuterium target (so that no subtraction of carbon data is necessary). The data for $\sigma_{0}$ were taken from Ref. [9]. The average deviation between the analyses using the carbon subtracted butanol or the liquid deuterium data in the denominator was $2.25 \%$ for recoil neutrons and $2.1 \%$ for recoil protons. For the latter, only data above $W=$ $1.6 \mathrm{GeV}$ were used for the comparison because for lower energies the detection efficiency for recoil protons [which cancels as long as Eq. (1) is used with the carbon subtracted butanol data] could not be determined precisely enough for a comparison to the results of Ref. [9] on an absolute scale.

The neutron data are in quite good agreement with the results from the BnGa model [22] and clearly rule out the MAID predictions [32]. The disagreement between measurement and MAID prediction can be easily traced to an unrealistically large contribution of the $N(1675) 5 / 2^{-}$state in the MAID model.

The helicity dependent cross sections $\sigma_{1 / 2}$ and $\sigma_{3 / 2}$ can be extracted as

$$
\sigma_{1 / 2}=\sigma_{0}(1+E), \quad \sigma_{3 / 2}=\sigma_{0}(1-E),
$$

from the asymmetry $E$ and the unpolarized cross section $\sigma_{0}$. For the latter the results from Ref. [9] were used. The results are summarized in Figs. 2 and 3. The systematic
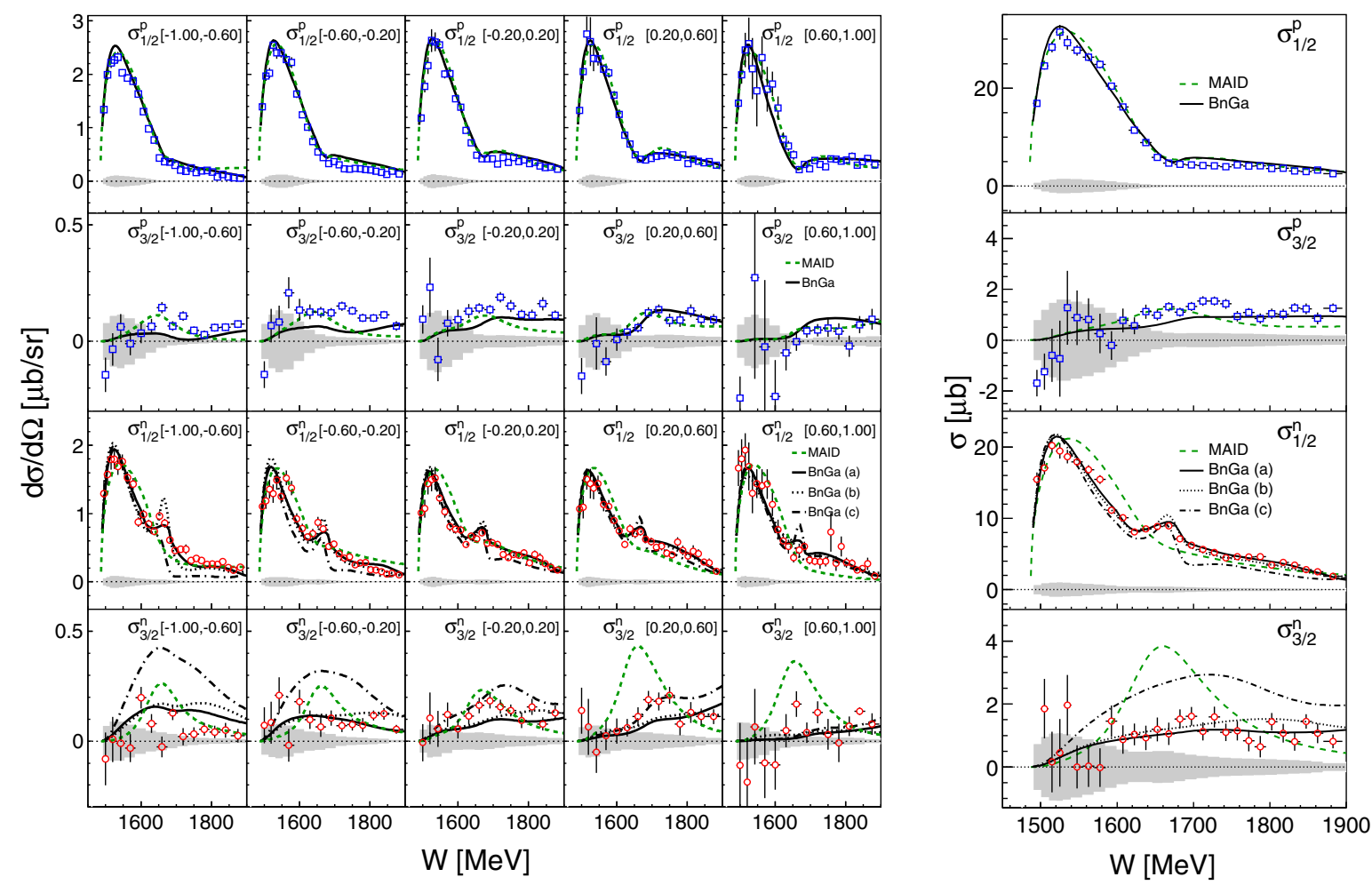

FIG. 2. Excitation functions for $\sigma_{1 / 2}$ and $\sigma_{3 / 2}$ for five $\cos \left(\theta_{\eta}^{\star}\right)$ bins [ $\cos (\theta)$ ranges given in figure]. Top two rows: results for $\gamma p \rightarrow p \eta$ (blue squares). Bottom two rows: $\gamma n \rightarrow n \eta$ (red circles). Gray shaded areas: systematic uncertainties. Curves: model predictions from MAID (dashed green line) [32], $\mathrm{BnGa}$ (a) (model version with interference in $S_{11}$ wave, solid black line) [22], BnGa(b) (model with narrow $P_{11}$ resonance with positive $A_{1 / 2}$ coupling, dotted black line) [22], and BnGa(c) (narrow $P_{11}$ resonance with negative $A_{1 / 2}$ coupling, dash-dotted, black line) [22]. Right hand side: total cross sections. 


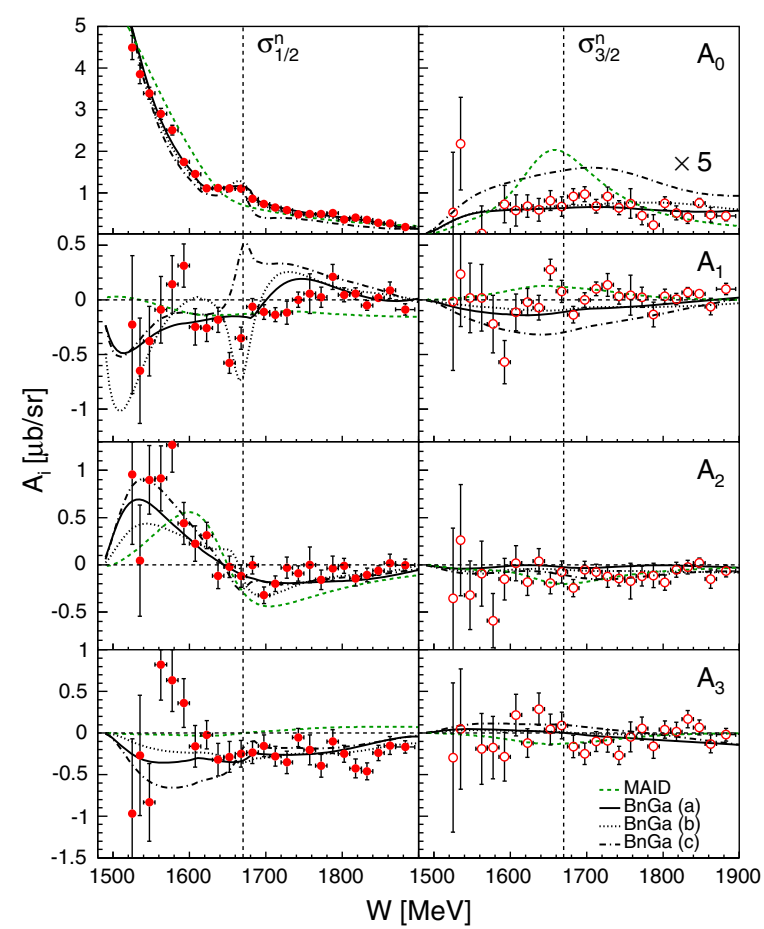

FIG. 3. Legendre coefficients of the angular distributions of $\sigma_{1 / 2}$ and $\sigma_{3 / 2}$ for the reaction $\gamma n \rightarrow n \eta$. Experimental results (red circles) and model predictions by MAID [32] and BnGa [22]. Same notation as in Fig. 2. The vertical dashed lines at $W=1685 \mathrm{MeV}$ indicate the position of the narrow structure. The results for $A_{0}, \sigma_{3 / 2}$ are up-scaled by a factor of 5 .

uncertainties for $E$ were propagated into Eq. (2). The overall systematic uncertainty for the scale of $\sigma_{0}$ from Ref. [9] is on the order of $7 \%-15 \%$. It is also possible to construct $\sigma_{1 / 2}$ and $\sigma_{3 / 2}$ directly from the data measured with the butanol target after subtraction of the carbon background without using input from the independent measurement of the unpolarized cross section. For the measurement with recoil neutrons, excellent agreement was found for all energies and center-of-momentum (c.m.) angles of the $\eta$, for recoil protons deviations occurred for $W<1.6 \mathrm{GeV}$ due to the known inaccuracies of the proton detection efficiency.

Figure 2 shows the excitation functions for five bins of $\cos \left(\theta_{\eta}^{\star}\right)\left[\theta_{\eta}^{\star}\right.$ polar angle in the photon-nucleon c.m. frame $]$ and the total cross sections in comparison to the predictions from the MAID [32] and BnGa [22] models. For protons and neutrons, contributions from the helicity- $3 / 2$ amplitude are small, which means that nucleon resonances with $J \geq 3 / 2$ contribute little. For the proton target, the $\sigma_{1 / 2}$ results are in good agreement with model predictions. The small $\sigma_{3 / 2}$ part is in reasonable agreement with model results. Details like the contribution of the $N(1720) 3 / 2^{+}$ state (a small enhancement with respect to the model results may be visible in the total $\sigma_{3 / 2}$ cross section in this energy range) will be subject to more refined partial wave analysis.
The results for the quasifree neutron establish that the narrow structure around $W \approx 1.67 \mathrm{GeV}$, listed as the tentative N(1685) state in RPP, appears only in the helicity$1 / 2$ part of the reaction. This means that it is almost certainly related to $J=1 / 2$ contributions $\left(S_{11}\right.$ and/or $P_{11}$ partial waves). Although excited nucleon states with $J \geq 3 / 2$ can also contribute to helicity $1 / 2$, it is unlikely that they contribute only to helicity $1 / 2$. The RPP [18] lists only one state up to excitation energies of $2 \mathrm{GeV}$ for which the helicity coupling $A_{1 / 2}$ is larger than $A_{3 / 2}$ (the $N(1875$ ) $3 / 2^{+}$for the proton, but even in that case within uncertainties $A_{3 / 2}$ could be larger). There is no example for such a state for which the helicity- $3 / 2$ contribution is negligible compared to helicity $1 / 2$. Since no trace of the structure is observed in helicity $3 / 2$, a contribution from $J \geq 3 / 2$ states is highly unlikely.

As mentioned above, a large contribution of the $N(1675)$ $5 / 2^{-}$state, as in the MAID model, was ruled out. In addition, the $\mathrm{BnGa}$ model with a narrow $P_{11}$ resonance with negative coupling disagrees with the experimental results, while the other two $\mathrm{BnGa}$ model versions give similar results. The angular distributions have been fitted with third order Legendre expansion to allow for a more detailed comparison to model predictions:

$$
\frac{d \sigma}{d \Omega}\left(W, \cos \left(\theta_{\eta}^{\star}\right)\right)=\frac{q_{\eta}^{\star}(W)}{k_{\gamma}^{\star}(W)} \sum_{i=0}^{3} A_{i}(W) P_{i}\left(\cos \left(\theta_{\eta}^{\star}\right)\right),
$$

where $q_{\eta}^{\star}$ and $k_{\gamma}^{\star}$ are the $\eta$ and photon momenta in the c.m. frame, respectively. The results are shown in Fig. 3. The $A_{1}$ coefficient for the $\sigma_{1 / 2}$ cross section is very interesting. An interference between a $P_{11}$ wave and the dominant $S_{11}$ wave results in $\operatorname{a} \cos \left(\theta_{\eta}^{\star}\right)$ term in the angular distribution, which is reflected in the $A_{1}$ coefficient. Depending on the sign of the interference term, a narrow $P_{11}$ resonance will result in a sharp positive or negative peak in $A_{1}$, as shown by the model curves in Fig. 3, while interference effects in the $S_{11}$ wave produce different patterns. The results clearly rule out the model version with a negative $P_{11}-S_{11}$ interference sign. However, the model results with a positive interference sign of $P_{11}$ and $S_{11}$ are more similar to the measured data than the predictions without the addition of a narrow $P_{11}$ state.

In summary, the double polarization observable $E$ and the related helicity dependent cross sections $\sigma_{1 / 2}$ and $\sigma_{3 / 2}$ were measured for the first time for the photoproduction of $\eta$ mesons on quasifree nucleons using a circularly polarized photon beam and a longitudinally polarized target. The measurement provided data of excellent quality, which are important input for future partial wave analysis of photoproduction of $\eta$ mesons off nucleons. Here, we report one striking finding about the nature of the narrow structure previously observed in the $\gamma n \rightarrow n \eta$ reaction. The results have unambiguously established that this structure is related to the helicity- $1 / 2$ amplitude and a comparison 
of the angular dependence to different model predictions favors a scenario with a contribution from a narrow $P_{11}$ resonance.

We wish to acknowledge the outstanding support of the accelerator group and operators of MAMI. This work was supported by Schweizerischer Nationalfonds (200020156983, 132799, 121781, 117601), Deutsche Forschungsgemeinschaft (SFB 443, SFB 1044, SFB/ TR16), the INFN-Italy, the European CommunityResearch Infrastructure Activity under FP7 programme (Hadron Physics, Grant Agreement No. 227431), the UK Science and Technology Facilities Council (ST/J000175/1, ST/G008604/1, ST/G008582/1,ST/J00006X/1, and ST/ L00478X/1), the Natural Sciences and Engineering Research Council (NSERC, FRN: SAPPJ-2015-00023), Canada. This material is based upon work also supported by the U.S. Department of Energy, Office of Science, Office of Nuclear Physics Research Division, under Awards No. DE-FG02-99-ER41110, No. DE-FG0288ER40415, and No. DE-FG02-01-ER41194 and by the National Science Foundation, under Grants No. PHY1039130 and No. IIA-1358175.

*Also at Dipartimento di Fisica, Università di Pavia, Pavia, Italy.

${ }^{\dagger}$ Present address: Institut für Kernphysik, FZ Jülich, 52425 Jülich, Germany.

Corresponding author bernd.krusche@unibas.ch

${ }^{\S}$ Present address: Department of Physics and Astronomy, Rutgers University, Piscataway, New Jersey, 08854-8019, USA.

[1] B. Krusche and S. Schadmand, Prog. Part. Nucl. Phys. 51, 399 (2003).

[2] B. Krusche and C. Wilkin, Prog. Part. Nucl. Phys. 80, 43 (2015).

[3] V. Kuznetsov et al., Phys. Lett. B 647, 23 (2007).

[4] I. Jaegle et al., Phys. Rev. Lett. 100, 252002 (2008).

[5] I. Jaegle et al., Eur. Phys. J. A 47, 89 (2011).

[6] F. Miyahara et al., Prog. Theor. Phys. Suppl. 168, 90 (2007).

[7] D. Werthmüller et al., Phys. Rev. Lett. 111, 232001 (2013).
[8] L. Witthauer et al., Eur. Phys. J. A 49, 154 (2013).

[9] D. Werthmüller et al., Phys. Rev. C 90, 015205 (2014).

[10] E. F. McNicoll et al., Phys. Rev. C 82, 035208 (2010).

[11] V. Kuznetsov, F. Mammoliti, V. Bellini, G. Gervino, F. Ghio, G. Giardina, W. Kim, G. Mandaglio, M. L. Sperduto, and C. M. Sutera, Phys. Rev. C 91, 042201(R) (2015).

[12] D. Werthmüller, L. Witthauer, D. I. Glazier, and B. Krusche, Phys. Rev. C 92, 069801 (2015).

[13] M. V. Polyakov and A. Rathke, Eur. Phys. J. A 18, 691 (2003).

[14] R. A. Arndt, Ya. I. Azimov, M. V. Polyakov, I. I. Strakovsky, and R. L. Workman, Phys. Rev. C 69, 035208 (2004).

[15] K.-S. Choi, S.-I. Nam, A. Hosaka, and H.-C. Kim, Phys. Lett. B 636, 253 (2006).

[16] A. Fix, L. Tiator, and M. V. Polyakov, Eur. Phys. J. A 32, 311 (2007).

[17] M. Shrestha and D. M. Manley, Phys. Rev. C 86, 045204 (2012); 86, 055203 (2012).

[18] K. A. Olive et al.(Particle Data Group), Chin. Phys. C 38, 090001 (2014).

[19] V. Shklyar, H. Lenske, and U. Mosel, Phys. Lett. B 650, 172 (2007).

[20] R. Shyam and O. Scholten, Phys. Rev. C 78, 065201 (2008).

[21] M. Döring and K. Nakayama, Phys. Lett. B 683, 145 (2010).

[22] A. V. Anisovich, E. Klempt, B. Krusche, V. A. Nikonov, A. V. Sarantsev, U. Thoma, and D. Werthmüller, Eur. Phys. J. A 51, 72 (2015).

[23] I. S. Barker, A. Donnachie, and J. K. Storrow, Nucl. Phys. B95, 347 (1975).

[24] I. Senderovich et al., Phys. Lett. B 755, 64 (2016).

[25] Th. Walcher, Prog. Part. Nucl. Phys. 24, 189 (1990).

[26] J. C. McGeorge et al., Eur. Phys. J. A 37, 129 (2008).

[27] H. Olsen and L. C. Maximon, Phys. Rev. 114, 887 (1959).

[28] C. Bradtke et al., Nucl. Instrum. Methods Phys. Res., Sect. A 436, 430 (1999).

[29] A. Starostin et al., Phys. Rev. C 64, 055205 (2001).

[30] A. R. Gabler et al., Nucl. Instrum. Methods Phys. Res., Sect. A 346, 168 (1994).

[31] D. Watts, in Calorimetry in Particle Physics, Proceedings of the 11th Internatinal Conference, Perugia, Italy, 2004, edited by C. Cecchi, P. Cenci, P. Lubrano, and M. Pepe (World Scientific, Singapore, 2005), p. 560.

[32] W.-T. Chiang, S. N. Yang, L. Tiator, and D. Drechsel, Nucl. Phys. A700, 429 (2002). 